\title{
Author Correction: Planar and van der Waals heterostructures for vertical tunnelling single electron transistors
}

\author{
Gwangwoo Kim', Sung-Soo Kim (10 2,12, Jonghyuk Jeon (10 2 , Seong In Yoon', Seokmo Hong ${ }^{3}$, Young Jin Cho ${ }^{4}$, \\ Abhishek Misra ${ }^{5,6}$, Servet Ozdemir ${ }^{5}$, Jun Yin ${ }^{5}$, Davit Ghazaryan ${ }^{5,7}$, Matthew Holwill (1) ${ }^{5}$, Artem Mishchenko (D) ${ }^{5}$, \\ Daria V. Andreeva ${ }^{8}$, Yong-Jin Kim", Hu Young Jeong (10 10, A-Rang Jang (1) 1,3, Hyun-Jong Chung (1) 4, \\ Andre K. Geim ${ }^{5}$, Kostya S. Novoselov ${ }^{5}$, Byeong-Hyeok Sohn ${ }^{2}$ \& Hyeon Suk Shin (1) 1,3,9,11
}

Correction to Nature Communications; https://doi.org/10.1038/s41467-018-08227-1, published online 16 January 2019

The original version of this Article contained an error in the spelling of the author Matthew Holwill, which was incorrectly given as Mathew Holwill. This has now been corrected in both the PDF and HTML versions of the Article.

Published online: 25 February 2019

Open Access This article is licensed under a Creative Commons Attribution 4.0 International License, which permits use, sharing, adaptation, distribution and reproduction in any medium or format, as long as you give appropriate credit to the original author(s) and the source, provide a link to the Creative Commons license, and indicate if changes were made. The images or other third party material in this article are included in the article's Creative Commons license, unless indicated otherwise in a credit line to the material. If material is not included in the article's Creative Commons license and your intended use is not permitted by statutory regulation or exceeds the permitted use, you will need to obtain permission directly from the copyright holder. To view a copy of this license, visit http://creativecommons.org/licenses/by/4.0/.
\end{abstract}

(C) The Author(s) 2019

\footnotetext{
${ }^{1}$ Department of Energy Engineering, Ulsan National Institute of Science \& Technology (UNIST), Ulsan 44919, Republic of Korea. ${ }^{2}$ Department of Chemistry, Seoul National University, Seoul 08826, Republic of Korea. ${ }^{3}$ Department of Chemistry, UNIST, Ulsan 44919, Republic of Korea. ${ }^{4}$ Department of Physics, Konkuk University, Seoul 05029, Republic of Korea. ${ }^{5}$ School of Physics and Astronomy, University of Manchester, Manchester M13 9PL, United Kingdom. ${ }^{6}$ Department of Physics, Indian Institute of Technology Madras, Chennai 600036, India. ${ }^{7}$ Department of Physics, National Research University Higher School of Economics, Staraya Basmannaya 21/4, Moscow 105066, Russian Federation. ${ }^{8}$ Department of Materials Science and Engineering, National University of Singapore, Singapore 117575, Singapore. ${ }^{9}$ Center for Multidimensional Carbon Materials, Institute of Basic Science (IBS), Ulsan 44919, Republic of Korea. ${ }^{10}$ UNIST Central Research Facilities (UCRF), UNIST, Ulsan 44919, Republic of Korea. ${ }^{11}$ Low Dimensional Carbon Material Center, UNIST, UIsan 44919, Republic of Korea. ${ }^{12}$ Present address: Carbon Composite Materials Research Center, Korea Institute of Science and Technology (KIST), Wanju 55324, Republic of Korea. The original article can be found online at https://doi.org/10.1038/s41467-018-08227-1. Correspondence and requests for materials should be addressed to K.S.N. (email: kostya@manchester.ac.uk) or to B.-H.S. (email: bhsohn@snu.ac.kr) or to H.S.S. (email: shin@unist.ac.kr)
} 